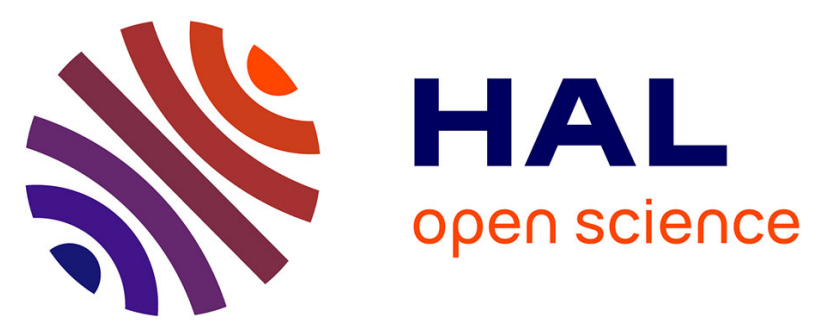

\title{
[Determinants of support for dementia patients in general practice: a qualitative approach based on an epidemiological cohort].
}

Agnes O. Engberink, Clément Pimouguet, Michel Amouyal, Olga Gerassimo, Gérard Bourrel, Claudine Berr

\section{To cite this version:}

Agnes O. Engberink, Clément Pimouguet, Michel Amouyal, Olga Gerassimo, Gérard Bourrel, et al.. [Determinants of support for dementia patients in general practice: a qualitative approach based on an epidemiological cohort].. Gériatrie et psychologie \& neuropsychiatrie du vieillissement, 2013, 11 (2), pp.157-67. 10.1684/pnv.2013.0413 . inserm-00835637

\section{HAL Id: inserm-00835637 https://www.hal.inserm.fr/inserm-00835637}

Submitted on 16 May 2014

HAL is a multi-disciplinary open access archive for the deposit and dissemination of scientific research documents, whether they are published or not. The documents may come from teaching and research institutions in France or abroad, or from public or private research centers.
L'archive ouverte pluridisciplinaire HAL, est destinée au dépôt et à la diffusion de documents scientifiques de niveau recherche, publiés ou non, émanant des établissements d'enseignement et de recherche français ou étrangers, des laboratoires publics ou privés. 
Titre : Déterminants de la prise en charge des patients déments dépistés dans une cohorte populationnelle : approche qualitative auprès de leurs médecins généralistes.

Title: Determinants of support for dementia patients in general practice: a qualitative approach based on an epidemiological cohort

Auteurs :

Oude Engberink Agnes 1,2,3

Clément Pimouguet ${ }^{4}$

Amouyal Michel ${ }^{1,3}$

Gerassimo Olga, ${ }^{1}$

Bourrel Gérard ${ }^{1,2,3}$

Berr Claudine ${ }^{3,5}$

${ }^{1}$ Département de Médecine Générale, Université Montpellier I

${ }^{2}$ EPSYLON (EA4556), Montpellier, F-34000

${ }^{3}$ Université Montpellier1, Montpellier, F-34000, France

${ }^{4}$ INSERM, ISPED, Centre INSERM U897-Epidemiologie-Biostatistique, F-33000 Bordeaux, ${ }^{5}$ France ${ }^{2}$ INSERM, Unité 1061, Montpellier, F-34093 France

\section{Auteur correspondant}

Claudine Berr

Inserm U1061,- Neuropsychiatrie : recherche épidémiologique et clinique

Hôpital de La Colombière,

BP 34493

34093 Montpellier Cedex 5, France

Tel: +33499614566

Fax: +33 499614579

Email: claudine.berr@inserm.fr 


\section{Résumé}

Différentes études internationales rapportent un sous-diagnostic important des patients déments. Une hypothèse expliquant ce sous-diagnostic serait l'attitude des médecins généralistes ne considérant pas la maladie comme une priorité. Le but de cette étude qualitative était de comprendre la variabilité de la prise en charge de la démence en explorant l'expérience vécue de médecins généralistes en situation clinique. Le recrutement des médecins généralistes a été réalisé à partir de cas incidents de démence, repérés dans l'étude de cohorte des 3 Cités. Des entretiens semi-directifs puis une analyse phénoménologique et pragmatique, ont permis de recueillir puis d'analyser la totalité du verbatim.

On trouve parmi les catégories émergentes, l'idée que pour les médecins, la maladie démence relève d'un problème de santé publique et que les identités personnelle et professionnelle du médecin sont déterminantes dans le retard au diagnostic et la variabilité des prises en charges. Dans un contexte de charge de travail accrue, le médecin privilégie les comorbidités sévères chez les personnes âgées compte tenu du flou nosologique et du doute sur l'efficacité thérapeutique des démences. L'approche phénoménologique permet de comprendre la variabilité des délais de prise en charge. 


\section{Abstract}

Background: The analysis of access to diagnosis and care pathway for dementia patients shows that the disease is not considered as a priority for the general practitioner (GP). Different studies have point out under diagnosis of dementia.

Purpose: The purpose of this qualitative study was to document the determinants of the diagnosis and management of dementia by GP.

Methods: Recruitment of GPs $(n=12)$ was made from incident cases of dementia who were identified during the follow-up of subject enrolled in the 3 Cities cohort study. A semistructured interview was conducted with an interview guide focused on the experience of the doctors. A phenomenological and pragmatic analysis, taking into account all the linguistic and extralinguistic evidence contained in the transcript was conducted.

Results: Several emerging categories (have been described: the doctors believe that the management of Alzheimer's disease is a public health problem and not an individual, the positioning of the GP in the care system is central. Determinants that influence the management are the identity of the physician, the impression of a fuzzy nosology, the finding of a therapeutic ineffectiveness, the priority given to severe co-morbidities and the workload of the general practitioner. However, the ordering of these categories according to the pragmatic phenomenological approach showed that the identity of the doctor, professional and personal, is at the origin of behavioral variability in their medical care.

Conclusion: In a context of increased workload, the GP favors the assumption of comorbidities in the elderly given fuzzy nosology of dementia and uncertainty about the therapeutic efficacy. The phenomenological approach allows understanding that the human identity of the doctor, personal and professional identity, is the major factor that influences its care attitude for demented patients. 
Mots-clés: démences, diagnostic, maladie d'Alzheimer, médecine générale, étude qualitative, analyse phénoménologique pragmatique

Key-words: dementia, Alzheimer's disease, diagnosis general practice, qualitative study, phenomenological-pragmatical approach.

\section{Point clefs :}

Les médecins généralistes considèrent la démence comme un problème de santé publique.

$\mathrm{Vu}$ leur charge de travail accrue, le flou nosologique des démences et leur doute dans l'efficacité des traitements, ils privilégient les comorbidités sévères et la prise en charge psychosociale de la dépendance.

L'approche phénoménologique pragmatique permet de comprendre la variabilité des prises en charge en considérant l'identité personnelle et professionnelle du médecin.

Considérer cette identité spécifique pour clarifier le rôle de chacun dans le système de soin pourrait améliorer le parcours de soin du patient dément.

Conflit d'intérêt : les auteurs n'ont aucun conflit d'intérêt en lien avec le texte. 
La démence est un trouble mental d'origine organique dont l'étiologie principale chez le sujet âgé est la maladie d'Alzheimer (MA). Elle touche principalement les personnes âgées, et conduit de façon inéluctable à une perte d'autonomie et une dépendance. Il existe aujourd'hui peu de moyens de prévention (prise en charge des facteurs de risque cardiovasculaire, nutrition, activités cognitives et physiques, équilibre affectif et social) et seuls des traitements symptomatiques d'efficacité limitée peuvent être proposés [1]. Il est important de noter qu'il n'existe pas de critères explicites et opérationnels du déclin cognitif ou du retentissement sur les activités de la vie quotidienne dans les recommandations de l'HAS [2]. Le diagnostic est donc laissé à l'appréciation subjective du clinicien alors que l'utilisation d'outils objectifs comme le test du Mini Mental Status Examination MMSE est loin d'être entrée dans la pratique courante des médecins généralistes.

En 2010 en France, 754000 personnes seraient atteintes de démence, dont 66,8\% en raison d'une MA et $72 \%$ des patients seraient des femmes. Ce chiffre représente $1,2 \%$ de la population totale, $7,9 \%$ de la population des plus de 65 ans et $2,8 \%$ de la population active [3]. Selon le baromètre santé Médecins Généralistes 2009 [4], 92\% des médecins généralistes interrogés déclarent avoir suivi au moins un patient atteint de MA dans l'année, 30,7\% en ayant accompagné plus de 10. Parmi ceux ayant un patient MA, 4 sur 10 déclarent connaître les recommandations de l'HAS pour le diagnostic et la prise en charge, cette connaissance étant associé à une propension plus importante à l'annonce du diagnostic. Huit médecins sur 10 déclarent orienter systématiquement vers un spécialiste. En revanche, seuls $15 \%$ déclarent utiliser des questionnaires pré établis pour évaluer la mémoire ou les troubles cognitifs.

Cela pourrait expliquer un diagnostic retardé au moment où des symptômes évidents voire des complications apparaissent. Ainsi, seulement une démence sur deux est diagnostiquée, voire même une sur trois au stade de début, avec un délai de deux ans entre les 
premiers symptômes et le diagnostic. Dans l'étude des 3 Cités [5], sur les 201 cas prévalents de démences repérés avec une démarche diagnostique active dans le cadre de l'étude, seuls $61 \%$ avaient consulté un médecin pour des troubles cognitifs et $32,9 \%$ avaient vu un spécialiste [6]. Un diagnostic précis a été annoncé au malade ou à sa famille dans $30 \%$ des cas. La plainte au médecin diminue nettement avec l'âge et le recours au spécialiste s'effondre après 80 ans passant de $55 \%$ à $20 \%$. Ces résultats ne sont pas spécifiques à la France. Ainsi dans une étude réalisée en Finlande, M. Löppönen et coll. [7] ont montré que 48\% seulement des sujets déments détectés dans le cadre de l'étude avaient été diagnostiqués par un médecin auparavant. Au début de la démence, un malade sur trois seulement était diagnostiqué, et même en fin de maladie, près d'un malade sur quatre était ignoré. Bien d'autres études ont montré une proportion élevée de cas non diagnostiqués dans la population en Suède [8], en Angleterre [9], au Canada [10] ou encore aux États-Unis [11]. Ces données sont corroborées par la «Facing Dementia Survey » [12,13], enquête d'opinion européenne réalisée par interviews dirigés dans 6 pays (France, Grande-Bretagne, Allemagne, Italie, Espagne et Pologne). Elle montre que selon les médecins, seuls 4 malades sur 10 consultent pour la première fois à un stade de démence légère, $54 \%$ à un stade modéré et $7 \%$ ne consultent qu'à un stade sévère.

Une enquête téléphonique récente montre que $69 \%$ de la population générale allemande souhaiterait participer à un dépistage précoce des démences et $2 / 3$ consulteraient leur médecin généraliste en cas de troubles cognitifs, loin devant les spécialistes [14]. Le médecin généraliste français occupe une place centrale dans le système de soin, renforcer par la réforme instituant le parcours de patients et obligeant celui-ci à désigner un médecin référent. Dans ce contexte le retard diagnostic mériterait d'être explicité. Est-il lié à un défaut de recours aux soins par les malades et leurs familles ou à un défaut de mise en place de procédures diagnostiques par le médecin généraliste en cas de plainte cognitive? 
La plupart des travaux de recherche quantitative ne permettent pas de comprendre vraiment la question du délai diagnostic. Les approches qualitatives explorant et analysant les attitudes et pratiques professionnelles dans les démences, sont peu nombreuses et restent à développer. A partir de cas incidents de démence, repérés au cours du suivi dans l'étude de cohorte des 3 Cités à Montpellier, une recherche qualitative a été conduite pour étudier les facteurs qui guident les attitudes des médecins généralistes, avec une approche phénoménologique apportant un éclairage nouveau sur les représentations, les affects, les habitus et les valeurs professionnelles des médecins. L'objectif de cette étude est de mieux comprendre la variabilité de la prise en charge de la démence en explorant l'expérience vécue des médecins généralistes en situation clinique. 


\section{Matériel et Méthodes}

Le centre de Montpellier de l'étude des 3 Cités a servi de cadre à ce travail. Cette étude de cohorte mise en place en 1999 avait pour objectif principal d'étudier les facteurs de risque vasculaire associés à la survenue d'une démence [5] . Le centre de Montpellier a suivi depuis cette date plus de 2000 personnes âgées de plus de 65 ans en recherchant, lors d'examens pratiqués environ tous les deux ans, l'existence de troubles cognitifs par un examen neuropsychologique et un examen médical standardisé. Ces suivis ont permis de repérer les cas incidents de démences apparus dans l'intervalle entre deux visites. Depuis le début de l'étude, pour tous les sujets présentant des troubles cognitifs faisant suspecter un diagnostic de démence, un courrier était adressé au médecin traitant désigné par le participant. Parmi les questions systématiquement posées aux participants, figuraient des questions sur les plaintes cognitives et la notion de recours à un médecin généraliste ou spécialiste devant ces plaintes. Ces données contemporaines du diagnostic de démence permettent de savoir si le sujet avait ou non consulté son médecin traitant ou un spécialiste pour une plainte cognitive. Elles ont permis de définir des cas index « consultants» ou « non consultants ».

\section{Choix des médecins interrogés.}

Les médecins interrogés étaient des médecins généralistes ayant un patient index (diagnostiqué au suivi à 10 ans de l'étude des $3 \mathrm{C}$ ). Une liste de 22 patients dont la démence venait d'être diagnostiquée dans l'étude a été établie (11 patients «consultants», 11 «non consultants »). Un courrier d'information sur le projet a été adressé et, après appel téléphonique, 12 praticiens ont accepté l'entretien (noté P1 à P12). Pour éviter une approche a priori, l'enquêteur (OG), médecin généraliste en fin d'étude, ne savait pas à quel groupe de 
patients était rattaché l'interviewé. La période d'entretiens s'est étendue du 30 mars au 1er juillet 2010 .

Méthode de recueil des données.

Notre choix était de ne pas partir de variables prédéterminées afin de laisser la possibilité d'émergence de facteurs nouveaux à partir d'une approche phénoménologique et pragmatique [15]. Pour explorer l'expérience vécue de chaque médecin [16], l'entretien individuel semi-directif [17] a été utilisé laissant plus de liberté d'expression au médecin et de possibilité de relance à l'enquêteur, plutôt que l'entretien de groupe, l'objectif se prêtant ici moins à une discussion collective.

Les rencontres ont eu lieu dans leur cabinet de consultation. Les médecins n'étaient pas rémunérés pour l'entretien. L'objectif de l'étude était présenté de la même façon à tous les praticiens. L'enregistrement a été fait avec un dictaphone digital après accord du médecin. Le matériau de recherche était le verbatim retranscrit le plus fidèlement possible. Une attitude empathique phénoménologique, c'est-à-dire facilitant le plus possible l'expression de l'interlocuteur [18], a présidé à l'entretien. Le questionnement était centré sur l'expérience propre de l'interviewé avec une grille d'entretien prévoyant des sous-questions de relance (cf. tableau 1).

\section{Méthode d'analyse}

L'approche phénoménologique permet la description du vécu à partir de catégories émergentes. La combinaison à la pragmatique de Peirce amène ensuite à restituer un sens

général à partir d'un ordonnancement logique des catégories obtenues [15,19]. Peirce a démontré que tout phénomène expérientiel est constitué de «trois catégories universelles » nécessaires et suffisantes pour l'expliquer : Catégorie de la Qualité (1) (sentiments, émotions), 
Catégorie des Faits (2) (action, constat, expérience), Catégorie de la Loi (3) (théorie, principe, habitus). Ces catégories présentent des relations de hiérarchisation entre elles (logique des relations) : (3) présuppose (2), qui présuppose (1) [20]. Cette configuration a permis de dire quelles catégories étaient les plus déterminantes pour organiser et donner du sens aux résultats.

Les étapes de l'analyse [21] sont les suivantes:

1/ lecture rapide, «flottante » permettant d'appréhender une ambiance du texte.

2/ lecture focalisée permettant un découpage en unité de sens et en thèmes.

3/ repérages des indices textuels et contextuels et mise-en-lien pour élaborer des catégories montant en généralité.

4/ caractérisation des catégories émergentes obtenues à partir des catégories sémiotiques universelles de Peirce selon (1), (2), (3).

5/ construction du sens par mise en ordre logique des catégories obtenues selon les relations qu'elles entretiennent entre elles.

6/ production d'une synthèse sous forme de proposition généralisante ou théorisante. 


\section{Résultats}

Au stade des 12 entretiens effectués, plus aucun indice nouveau n'est venu enrichir l'information déjà obtenue. Le phénomène de saturation étant probablement atteint, il n'a pas été nécessaire de relancer d'autres médecins. L'analyse des énoncés a fait émerger des catégories qui, mises en lien, ont pu dégager des catégories signifiantes plus larges dénommées catégories conceptualisantes.

\section{Les démences sont considérées par les médecins comme un «problème de santé publique » dépassant le cadre de la médecine générale.}

Cette expression utilisée par des généralistes montre leur sentiment que le phénomène étudié est multidimensionnel, qu'il «ne pouvait être pris en charge par une seule personne» mais par un «système» organisé. Derrière cette notion, il y a celles d'équipe pluridisciplinaire, de réseau pluri-professionnel médico-social ou social dans lequel l'aidant naturel a sa place. Pour certains, l'institutionnalisation fait partie des réponses organisées face au phénomène des démences. $\mathrm{P}$ 8: «s'il n'y a plus de réseau social il y aura des problèmes ». Les médecins notent l'importance de la proximité du spécialiste ou de réseaux.

P10: «on a la chance d'avoir un neurologue pas loin d'ici qui fait partie du réseau mémoire.. » $\mathrm{P} 3$ : «ce n'est pas un problème médical, c'est un problème d'organisation ». Certains anticipent ces difficultés en organisant des Maisons de Santé pluridisciplinaires : «j'ai construit cette structure pour prendre en charge ces... »(P5). $\quad$ Un souhait d'avoir un dépistage systématique ciblé plutôt qu'un dépistage individuel opportuniste est exprimé : «moi j’aimerais qu’on réfléchisse à un dépistage...par exemple définir un âge où le pic est plus important...en même temps cela permettrait aux gens de ne pas le vivre comme un drame... »(P7). 


\section{La difficulté de l'abord du problème et de l'annonce :}

Certains médecins évoquaient leurs difficultés pour annoncer la maladie : «ce n'est pas évident d'annoncer ce qui arrive. Aller annoncer à quelqu'un qui hein «monsieur vous perdez un peu la boule! Allez lui expliquez ça» (P11); $\mathrm{P} 7$ : «vous dites à quelqu'un : «il y a un problème...démence vasculaire...ça fout pas la panique» si je dis «votre mari a un Alzheimer » je vois la patiente paniquer...». «On a une amplification d'un syndrome panique que le patient ressent ». Mais pour d'autres, ce n'est pas une difficulté : «aborder le problème ne me gêne pas puisqu'on n'a pas autre chose à proposer » (P8) ; P6 trouvait normal de le faire : «Ben, c'est notre rôle, hein! si chaque fois qu'il y a des choses violentes comme le Sida, les cancers et tout... ».

\section{Une orientation et un délai d'accès aux soins spécialisés dépendant de l'origine de l'alerte :}

Lorsque c'est le patient qui se plaint d'un trouble cognitif, l'orientation est plus facile, mais les éléments du diagnostic ne sont pas suffisants : «la plainte spontanée oriente vers un trouble bénin» $(\mathrm{P} 4) ; \mathrm{P} 8$ : «Mais les patients ont globalement plus de plaintes physiques » et « sa grosse plainte, [...] c'est pas sur le plan cognitif, c'est qu'elle peut plus marcher ». La plainte vient aussi souvent de l'entourage $(\mathrm{P} 8, \mathrm{P} 9, \mathrm{P} 2)$ : «c'est souvent l'entourage qui en parle... quand la famille vient me dire...c'est important de prendre en compte ».

Le déni des troubles ou une anosognosie sont aussi, un obstacle dans toutes les étapes. P4 : «comment faire comprendre à quelqu'un en dénégation de voir un spécialiste ». A la question : «est-ce qu'elle s'était plainte » $\mathrm{P} 1$ répond : « ah non elle ne risquait pas, elle ne s'en rendait pas compte. Elle était souriante en permanence, donc bon... ». 
L'isolement est aussi un obstacle à tous les niveaux : «on envoie au neurologue, mais faut déjà détecter...mais ça c'est pas très évident parce que la plupart du temps ces gens sont seuls $\gg(\mathrm{P} 3)$.

L'orientation vers le spécialiste peut être retardée par le patient ou l'entourage : P11 : «décider les gens à aller voir le spécialiste, c’est ça le plus dur !»; P10 : «Mme B par exemple elle n'est pas traitée du tout puisqu'elle refuse complètement. Sa famille ne souhaite pas qu'elle consulte un neurologue... » $\mathrm{P} 4:$ : le problème c'est que quand ils sont d'accord c'est tôt, et quand ils sont pas d'accord c'est tard. »

Les prises en charges multiples dans le parcours de soin retardent la prise en charge pour les troubles cognitifs: $\mathrm{P} 2$ : «elle avait son cardio....elle avait pas vraiment de médecin généraliste. Donc je l'ai prise en charge un peu tard»; $\mathrm{P} 4$ «chez cette dame, il y avait plein de choses qui étaient intriquées...et elle était suivie par beaucoup de gens... un homéopathe. ..Les troubles cognitifs sont passés sur sa grosse anxiété depuis le décès de son mari ». Habitué au suivi sur le long terme, le temps du généraliste peut apparaitre en décalage par rapport au début de la plainte ou des troubles : «si l'on a des troubles cognitifs, on commence tout de suite, tout de suite, enfin, dans les semaines ou les mois qui viennent on prend des rendez-vous... » (P3). Mais, lorsque $\mathrm{P} 4$ a un doute, il n'hésite pas à orienter vers le spécialiste: «lorsque j'ai un doute sur un trouble cognitif je demande rapidement une consultation spécialisée ».

Enfin, l'impression que les spécialistes de ville éprouvent des difficultés aux premiers stades des démences a aussi son influence sur l'attitude des médecins. P7 : «on nous dit: il faut prendre au début » mais c'est quand le début? Une de mes patientes m'avait inquiété et ben le neuro a dit : on va se donner un peu de temps...on va voir venir » «je ne suis pas sûre que les spécialistes aient vraiment la réponse au début». P8: «je l'ai envoyé chez le 
neurologue qui m'a dit c'est un début d'Alzheimer mais je n'en suis pas sûr, donc après on l'a suivi de près ».

\section{Des limites liées à la charge de travail :}

La prise en charge apparait limitée par la charge de travail des médecins qu'il s'agisse de manque de temps ou de difficultés liées à la charge affective. La lourdeur de la prise en charge médicale et psycho-sociale est mise en avant par les médecins, $\mathrm{P} 4$ : «quand ils ne veulent pas voir le spécialiste, je me lance dans les tests même si je n'ai pas beaucoup de temps...mais après avoir fait le test je peux rien prescrire ». $\mathrm{P} 3$ : «ça nous prend beaucoup de temps et on a que très peu de temps ». $\mathrm{P} 5:$ : à 22 euros on ne peut pas faire une consultation d'une heure! ». P4 : «oui, ça fait beaucoup de démarche sociales... oui ça c'est difficile, on ne sait jamais à quelle porte s'adresser ».

De la charge en termes de temps à l'épuisement, il n'y a qu'un pas. P7 : «parce que émotionnellement si on nous fait tout porter, on aura un épuisement trop rapide et on deviendra des mauvais soignants »; P7: «Alors je n'ose imaginer mes confrères en campagne! Ils vont péter les plombs!». P11: «Si j’avais le temps...ce soir je finis à 21h, avant hier soir c'était $22 \mathrm{~h} 30$ !...« quand j'entends dire qu'un quart des médecins a pensé au suicide ?».

Le positionnement du médecin dans le système de soin caractérisé par une approche globale biopsychosociale.

Devant une plainte cognitive, les médecins disent explorer d'abord le contexte général clinique, physique, psychologique et sociale, caractéristique de l'approche holistique du généraliste : «pour débrouiller au démarrage » $(\mathrm{P} 4) . \mathrm{P} 4:$ «Oui, tout à fait on lance les 
examens souvent... on fait un bilan (vitamine B12, hypothyrö̈die, scanner, etc); P1 : «quand il commence à y avoir une plainte, c'est bien de faire un « état des lieux».

A cela s'ajoute la conscience de leur responsabilité sociale: $\mathrm{P} 7:$ : on ne peut pas laisser quelqu'un dans un immeuble où il y a du gaz!», «c'est ce genre de personne seule qu'il faut cibler ». Ils ont une place particulière dans la gestion de l'entourage. $\mathrm{P} 7:$ : le généraliste, il peut pas tout faire. Moi je gère le malade mais je gère beaucoup la famille. Je pense que le neuro, il va gérer le malade et moins la famille »

L'idée est ancrée dans la pratique que le médecin s'occupe du contexte clinique général et médico-social et moins du diagnostic : P7 : «On est complémentaire ! Nous sur la prise en charge familiale et sociale quelque part, l'hôpital pour l'identification du syndrome réel...le traitement... ». Mais les médecins généralistes s'estiment globalement au cœur du problème («chef d'entreprise », «coordonnateur » (P5), «responsable d'une équipe »), bien placés pour organiser «tout le travail d'encadrement» (P8), sans en avoir le temps et les moyens. Ils ont du mal à coordonner tous les intervenants, dans un contexte de comorbidités lourd. $\mathrm{P7}$ : «on ne peut pas dire que le généraliste est le pilier de tout »

\section{L'influence de l'identité personnelle et professionnelle du médecin dans le type de prise en charge.}

Elle donne lieu à une grande variabilité de prises en charge dépendant :

- de «l'être-médecin », de sa conception du métier, de son mode d'exercice seul ou dans des structures de type pôle de santé, de son niveau d'utilisation de l'informatique, à la ville ou à la campagne : P5 : «l'appréhension du problème par chaque médecin est lié à leur mode de pratique...».P7 : «Alors je n’ose imaginer mes confrères en campagne! ils vont péter les plombs!» devant la surcharge de travail et l'isolement. $\mathrm{P} 9$ : «suivant que je les vois à domicile ou au cabinet c'est pas pareil.. » 
- de l'implication qu'il développe dans un métier et une pathologie à forte tonalité affective : P7 : «Alors moi personnellement je vis très mal la perte d'autonomie », celle des gens qu'il aime. L'étude montre un médecin «optimiste» (P11), «c'est un papy adorable» ou «fataliste avec un sentiment d'impuissance »(P7).

\section{Constat de flou nosologique avec une connaissance de la pathologie variable selon les praticiens laissant une impression de doute.}

P1 émet des doutes sur la pertinence en pratique d'une classification des démences : «on appelle Alzheimer beaucoup de choses qui ne sont pas forcément la même chose », " on met des cases, mais je ne suis pas certain que ce soit pertinent ». Le symptôme «perte de mémoire » n'est pas perçu comme un signe d'alerte mais plus dans un contexte global : P9 P10: « si c'est un trouble de mémoire je me précipite pas tout de suite...ça dépend du contexte pathologique associé ».

Pour la plupart des médecins, l'approche clinique est perçue davantage dans sa dimension psychosociale, comportementale, comme le repérage de «petites choses », un changement d'habitude, de comportements, dans une pathologie s'accompagnant d'anosognosie. S'y ajoute un problème de fiabilité d'outils d'évaluation clinique à disposition des médecins généralistes : $\mathrm{P9}$ : «au niveau réel de la pathologie c'est difficile pour moi de l'apprécier objectivement »; 4 : « pour catégoriser on n'a pas les outils suffisants....assez précis pour aller pile au bon endroit ».

\section{Des traitements peu efficaces et éthiquement discutables.}

Les médecins expriment, à propos des traitements, qu' «avec du recul ça change peu l'évolution ». Etre non-prescripteur ne les dérange pas en raison du peu d'effet constaté des traitements. $\mathrm{P} 3$ : «ça ne me dérange pas car de toutes façons ce n'est pas très efficace ». 
P8 :«le seul patient étiqueté Alzheimer, que j’ai eu vraiment avec un effet important...euh, je sais même pas si...».

Cependant pour $\mathrm{P} 4$, le fait de ne pouvoir prescrire est un frein au diagnostic: «quand ils ne veulent pas voir le spécialiste, je me lance dans les tests même si je n'ai pas beaucoup de temps...mais après avoir fait le test, je ne peux rien prescrire ». La difficulté d'évaluer ces traitements s'ajoute au doute sur l'efficacité. P3 : «c'est un peu comme différents traitements contre euh, l'arthrose : si on n'avait pas donné, est-ce que ce serait pire?»

Ils s'impliquent affectivement et s'interrogent sur la pertinence de prescrire des médicaments qui pourraient prolonger la vie de personnes dépendantes. $\mathrm{P} 7$ : «de gens qu’on aime et que l'on va devoir prendre en charge jusqu'au bout» ou «Quel avantage de les maintenir de soixante quinze ans à quatre-vingts si ces années là t'as été en dégradation...est-ce que la société en imposant la vie à des gens c'est pas une maltraitance?».

Enfin, leurs choix vont vers des prises en charge qu'ils souhaiteraient «plus globales», interdisciplinaires : «entourer, stimuler, c'est ça qui est important ».

\section{L'existence de comorbidités sévères au premier plan de la prise en charge}

Même s'ils ont connaissance des troubles cognitifs, les médecins priorisent la prise en charge de comorbidités avérées sur un trouble cognitif perçu incertain. A la question : «le diagnostic a t-il modifié votre prise en charge ? »P3 répond : «je dirais que non car c'est une patiente qui a de gros problèmes cardio-vasculaires, donc moi j'étais plus axée là-dessus et maintenant une insuffisance rénale sévère ...»; P8 : «elle a plus que des troubles cognitifs cette femme, euh, elle tombe, voilà elle a fait des accidents vasculaires cérébraux», «sa grosse plainte c'est sur le plan physique...c'est qu'elle peut plus marcher ». Le souci de la prise en charge est centré sur le contexte pathologique entourant la démence, des comorbidités 
qui sont de leur responsabilité de généraliste est au centre de leur préoccupation. P7 : «Mais bon...dans l'année il y a eu le décès de son mari...un cancer du sein...chirurgie...radiothérapie...eu bon c'est quand même très perturbant...elle me fait des troubles mnésiques... elle avait arrêté ses traitements d'hypertension. Donc attention, risque vasculaire...ça fait beaucoup de choses ! »; P9 -P10: «si c'est un trouble de mémoire je me précipite pas tout de suite...ça dépend du contexte pathologique associé»; $\mathrm{P} 10$ : «une fois sur deux ça commence par un état dépressif ».

\section{Gestion difficile de l'entourage et de ses représentations}

Les superlatifs concernant cette charge sont édifiants P1: «catastrophique, compliqué... », P5 «c'est un combat», avec la problématique d'une éventuelle entrée en institution : P7 «envisager de mettre un patient en institution avec l'entourage est un travail ». $\mathrm{P} 3$ : « on sait que ça va être une bataille avec les proches ».

Le souci de la santé du conjoint entre aussi en jeu : «ce n'est pas la peine que l'autre plonge aussi ».

A contrario l'entourage peut être informant ou aidant naturel : P9 : « elle est bien aidée au niveau familial, ça compte beaucoup... sinon ce serait difficile ».

Ces deux positions ambigües de l'entourage reflètent les difficultés vécues par l'entourage d'une maladie stigmatisante.

\section{Restitution du sens par ordonnancement logique des catégories obtenues selon Peirce}

[20]: C'est la dernière étape du processus d'analyse de la signification. Il s'agit d'une synthèse ordonnée de toutes les catégories et de leurs dimensions donnant le sens général du phénomène étudié. 
Au total, les médecins généralistes pensent que la démence est un problème de santé publique (3) malgré leur positionnement central dans le système de soin (3); cela nécessite une organisation pluriprofessionnelle (2) devant la charge de travail (2) qui rend la coordination du parcours de soin et la prise en charge difficile (1)

L'identité personnelle et professionnelle du médecin (3) (caractérisé par une approche globale biopsychosociale), devant le flou nosologique et thérapeutiques (3), induit des modalités de prises en charge variables (2) (orientation vers un spécialiste, moment d'annonce diagnostic, priorisation des comorbidités, gestion de l'entourage) et des vécus différents (1).

\section{Comparaison aux informations recueillis sur le recours au médecin et la prise en charge} dans l'étude 3C.

Les propos des médecins sur leur cas index ont été secondairement comparés aux données recueillies par le centre $3 \mathrm{C}$ directement auprès des patients sur les plaintes cognitives, le recours à un médecin (notion de consultation pour troubles cognitifs), le diagnostic et la prise en charge. Ces deux sources de données se sont avérées complémentaires mais aussi contradictoires.

La répartition initiale en deux groupes en fonction de la notion d'une consultation pour troubles cognitifs, ne parait pas pertinente si l'on prend en compte les faits sans considérer l'intentionnalité des médecins (P3 évoque un retard «volontaire»). Pour les médecins dont le cas index n'a pas consulté, l'absence de diagnostic n'était la cause d'une absence de prise en charge que dans 1 cas sur 5 (patiente isolée et en institution). La plainte spontanée des patients n'est pas le déterminant de la prise en charge. Ce critère de recours aux soins tel qu'il peut être recueilli dans un cadre épidémiologique peut être tempéré à l'aune d'une étude qualitative. 


\section{Discussion}

Les sentiments issus du discours des médecins montrent leur impuissance, leur solitude et un ressenti d'inefficacité personnelle qui vont de pair avec le surmenage. Les médecins interrogés déclarent un souci d'efficience et un besoin de travailler en réseau. Plus que le sous diagnostic rapporté dans de nombreuses études dans le monde, c'est le délai de prise en charge qui est au cœur du problème. $\mathrm{Vu}$ la complexité ressentie des troubles cognitifs, les médecins se donnent le temps pour faire le diagnostic.

Parmi les éléments apportés par les entretiens, deux axes sont apparus plus importants :

- pour les médecins, la procédure diagnostique ne semble que rarement pertinente (état de santé précaire, comorbidités) ou applicable (déni des troubles, isolement ou absence d'informant, refus de la famille), et il est difficile de poser un diagnostic formel.

- de nombreux éléments sont identifiés expliquant le retard pour initier ou envisager cette procédure : priorisation des urgences médicales pour le médecin, flou nosologique, temporisation des spécialistes, contexte psychologique et conditions de vie du sujet. L'expertise collective de l'Inserm [22] concernant l'analyse de l'accès au diagnostic et du parcours de soins des MA, avait indiqué que la maladie n'est pas considérée comme une priorité pour le généraliste. Ces travaux mettaient en avant un certain nombre de déterminants comme la confusion entre démence débutante et vieillissement, la fréquence de comorbidités organiques et sensorielles, un contexte culturel défavorable, une représentation sociale catastrophique, un manque d'informants dans le cas de malades isolés, ou un déni des troubles. La coordination du parcours de soin des patients déments s'avère complexe malgré la place centrale du médecin généraliste (intervenant multiples, déni des troubles, contexte psychosocial et comorbidité du patient). Malgré cela, le médecin généraliste garde une position favorable pour initier la démarche diagnostique. Nos travaux sont en accord avec une large part de ce rapport d'expertise hors référence au contexte culturel. Les grandes catégories 
de déterminants connus sont retrouvées (déni, reconnaissance de la maladie, comorbidités, caractéristiques sémiologiques, attitudes des médecins, parcours de soin), mais l'étude qualitative en donne une compréhension plus proche de la réalité vécue en pointant l'importance de chacune par rapport aux autres. Les catégories émergentes de l'étude apportent un autre éclairage sur les déterminants que des rubriques classificatrices et peuvent orienter le sens différemment en les nuançant.

\section{Procédure diagnostique}

Plutôt qu'une confusion entre démence et vieillissement alléguée dans les études, notre analyse montre que la difficulté est de repérer une démence dans le cadre non pas d'un vieillissement normal, mais chez des patients âgés ayant un vieillissement pathologique avec des comorbidités. Les généralistes s'en remettent habituellement aux spécialistes sans aucune difficulté à déléguer. Et même dans ce cas, leur impression de flou nosologique est souvent renforcée par l'attitude attentiste des confrères spécialistes préférant temporiser, au risque de ne pouvoir revoir le patient par la difficulté d'accès aux soins (rendez-vous, transport, nécessité d'un accompagnant) ou sa résistance à consulter à nouveau. Ces faits soulignent l'importance du moment le plus opportun pour proposer la consultation de diagnostic.

Par ailleurs, les liens affectifs forts entretenus avec certains patients pourraient interférer avec l'intuition clinique du médecin, désemparé par le doute nosologique et sur l'efficacité thérapeutique. Là encore, le médecin donne la priorité aux comorbidités pour lesquelles il a des solutions thérapeutiques à proposer. Habitués à l'approche globale des problèmes, ils attachent tous de l'importance au contexte, en particulier aux pathologies vasculaires associées dont ils connaissent la place dans les pistes de prévention des démences. L'exemple du couple dépression-déclin des performances cognitives est un des plus difficiles à gérer pour la plupart des médecins : symptôme des déficits sensoriels du vieillissement, 
comorbidités angoissantes, iatrogénie des psychotropes, début de démence ; il faut du temps pour apprécier un diagnostic différentiel souvent intriqué. Si bien que, dans le cas de sujets indemnes de comorbidités, plus que des caractéristiques sémiologiques jugées floues, ce sont les «petites choses de la vie », un changement de comportement dans la quotidienneté sociale familière au médecin, qui l'oriente vers une suspicion de troubles cognitifs. Enfin, certains médecins estiment qu'un dépistage organisé systématique sur une tranche d'âge de population ciblée serait une solution de nature à leur faciliter la tâche.

\section{Retard dans la procédure diagnostique}

Les données de la «Facing Dementia Survey » [12] montraient que le délai entre la première consultation pour des troubles cognitifs et le diagnostic était de 24 mois en France. Peut-on mieux comprendre ce délai au diagnostic et à la prise en charge et comment le réduire? Concernant l'enregistrement de la plainte, l'étude montre que le généraliste oriente ses doutes en fonction de la présence d'un informant fiable. L'isolement du patient est en effet un facteur connu de sous-diagnostic par les médecins généralistes [23]. Lorsqu'il doit poser lui-même la question, le généraliste le fait selon sa représentation du métier, les valeurs qui s'y attachent et son degré d'implication. Considérant les motifs de délai relevés dans cette étude (déni, accès aux soins difficile, consultation spécialisée trop tôt, comorbidités urgentes, rendez-vous espacés) et leur attribution plutôt à l'identité des généralistes qu'à ses compétences, ce délai paraît difficilement compressible. Il est intéressant de comparer ce délai entre les pays, en effet en Allemagne il est de 10 mois, alors que les médecins généralistes allemands peuvent prescrire les traitements spécifiques [24].

En arrière plan de la question du diagnostic, il y a un principe empirique et d'efficience retrouvé en médecine générale énoncé ainsi par un médecin : « est-ce que ça vaut la peine de diagnostiquer quelque chose lorsqu'on n'a pas de traitement dont l'efficacité est 
démontrée ? ». La position de l'HAS sur le Service Médical Rendu faible des anticholinestérasiques semble leur donner raison aujourd'hui [25] En revanche, comme le souligne le dernier rapport mondial sur la MA [26], le diagnostic précoce pourrait apporter un bénéfice au patient de par les possibilités, entre autres, de mise en place d'un plan d'aide personnalisé (prévu dans le plan Alzheimer 2008-2012) ou d'une protection juridique.

Pour les médecins interrogés, la démence n'est pas une urgence et s'inscrit dans un suivi au long cours. La notion de temps du généraliste est liée à sa pratique. Confronté au suivi des maladies chroniques, il est habitué à une gestion de l'incertitude des stades de début, ce qui l'incite à une démarche intuitive et à temporiser, puisqu'il reverra le malade pour le suivi des comorbidités.

L'étude montre que la proximité au long cours du patient et de son entourage (caractéristique psychoaffective propre au métier de généraliste) peut l'amener à adopter une attitude de «sympathie » lorsqu'il ressent lui-même la «violence » de la maladie. Ainsi, le profil de certains déments «agréables et souriants» qui font partie «des gens que l'on aime » peut être un obstacle à l'écoute et au diagnostic. Cette relation particulière induit que certains médecins (les plus âgés) se projettent «comme s'ils étaient à la place des malades ». Ne pas prendre en compte cette donnée ferait dévier l'analyse vers le critère de manque de compétence souvent évoqué, lié à une formation initiale insuffisante des médecins [22].

La question de la charge de travail est un déterminant important pour l'ensemble des généralistes de l'étude, gérant à la fois le médical et le médico-social, déplorant un manque de reconnaissance en rapport. Leur charge de travail est variable suivant les modes d'exercice et l'existence d'un réseau médical et/ou médicosocial. Dans un contexte de pénurie de médecins, le médecin généraliste opère des choix et hiérarchise les prises en charge en fonction de l'urgence qu'il définit. En outre, l'étude montre que les médecins se protègent d'un épuisement (burn-out) qui les guette. Ils se sentent en capacité d'être «chef d'orchestre », 
mais éprouvent des difficultés dans la continuité des soins (changement de médecin-traitant, institutionnalisation/ hospitalisation des patients, prises en charge par des spécialistes divers sans réelle coordination). Ils déplorent le manque d'organisation du système de soins actuel dans la définition des rôles de chacun, véritable obstacle à un parcours de soin coordonné qui leur échappe mais dont ils sont un pilier central assumant un rôle de « débrouillage » global, d'évaluation du « contexte des pathologies associées ».

A notre connaissance, seulement deux études relatives aux attitudes des médecins généralistes face au diagnostic des démences ont été publiées dans la littérature internationale. La première synthétise les résultats de 3 projets de recherche qualitatifs menés entre 2003 et 2006 en Australie [27] et incluant 24 généralistes. Ces projets questionnaient leur expérience et leur représentation du diagnostic de démence dans le but d'expliciter les raisons du retard diagnostic en soins primaires. Douze médecins ont participé à des entretiens de groupe, 13 à des entretiens semi-dirigés en profondeur. Une analyse thématique à permis d'isoler 4 catégories identiques aux nôtres (reconnaissance de la démence, vision holistique, relation avec la famille et le patient, les traitements médicamenteux). Pour les MG australiens reconnaître la démence s'accompagne d'un délai conscient du à leur crainte de stigmatisation et leur doute sur l'intérêt de l'annonce précoce d'une maladie dont les traitements sont inefficaces. Seule une approche holistique du patient, considérant leurs comorbidités physiques, mentales et problématiques sociales, leur donne des arguments pour poser un diagnostic formel de démence dans le but d'améliorer leur accès aux soins ou leur maintien à domicile.

Enfin, un travail qualitatif récent français [28] étudiant les représentations sociales de $24 \mathrm{MG}$ enseignants parisiens et leur influence dans leur prise en charge des patients atteints de maladie d'Alzheimer conclut aussi que ce n'est pas par manque de compétence que les MG ne diagnostiquent pas, mais parce que pour eux il s'agit plutôt d'une maladie de l'autonomie 
sans traitement spécifique. Le risque de stigmatisation rend l'annonce du diagnostic secondaire et est laissée au spécialiste. Dans ce contexte, leur objectif est de soutenir patient et famille pour permettre un maintien au domicile ou pour la préparation d'une institutionnalisation.

Ce qu'apporte l'approche phénoménologique et pragmatique employée dans notre travail est une mise en ordre des catégories obtenues. L'utilisation de la sémiotique de Peirce désigne le déterminant le plus important dans sa capacité à renseigner le phénomène «retard/délai au diagnostic ». Dans notre enquête, la catégorie déterminante «penser le diagnostic d'Alzheimer comme un problème de santé publique » (théorie) relègue les catégories concernant la position du médecin généraliste dans le système de soin, l'approche globale, le souhait d'un dépistage organisé, la vision «maladie de l'autonomie », comme des propriétés (sous-catégories) qui la documentent. La catégorie relevant de l'identité personnelle et professionnelle explicite les types de comportement et leur variabilité. Le terme d'identité relève à la fois ce qui différencie le sujet dans sa relation à l'autre et qui résiste à ses variations dans le temps, et aussi ce qui renvoie à une appartenance collective, ici professionnelle [29]. Or, l'étude a montré que c'est l'identité du médecin qui guide ses comportements à partir de ses représentations, valeurs, principes généraux empiriques. En terme de stratégie de changement des comportements, ces résultats orienteraient vers la nécessité de prendre compte l'identité et le rôle des médecins généralistes dans le système de soin, plutôt que d'orienter vers un manque de compétence et une nécessité de formation.

\section{Forces et limites de l'approche}

Nos résultats ont été obtenus à partir de l'approche phénoménologique qui exige une familiarisation avec une sémantique perçue comme compliquée, ainsi qu'une habileté particulière à mener, un questionnement centré sur le vécu. Elle permet de documenter de 
façon plus fine et de nuancer les résultats d'enquêtes quantitatives leur donnant du sens, afin de limiter des jugements hâtifs et des stratégies inadéquates. Cette méthode montre l'écart entre l'information déclarative et celle émergeant de la réalité vécue. Le recueil par entretien semi-structuré a aidé à garder le fil conducteur de la recherche en laissant une souplesse de travail. La manière dont ce guide d'entretien a été conduit n'a pas toujours évité les pièges par inexpérience : défaut de relance, errance, suggestions. Mais malgré le nombre restreint de médecins interrogés, la saturation des données a été obtenue et un consensus a émergé des réponses. Le choix de prolonger l'approche descriptive phénoménologique par la pragmatique de Peirce répond à un souci de rigueur permettant la prise en compte tous les «indices » disponibles dans le matériau textuel, linguistique (mots, sémantique, rhétorique, figures de style) ou extralinguistiques (éléments des différents contextes).

\section{Conclusion}

L'apport de ce travail est un éclairage plus pertinent sur le délai du diagnostic et de la prise en charge des démences. La dimension identitaire des médecins, individuelle et professionnelle à l'origine de la variabilité des représentations et des comportements, est l'élément fondamental expliquant ce délai. Cette identité spécifique devrait être prise en compte dans une organisation du système de soin en réseau avec un parcours coordonné dans laquelle chacun ait un rôle mieux défini dans la prise en charge de la maladie d'Alzheimer. Plus que dans le diagnostic, ils éprouvent des difficultés dans la gestion de la relation au malade dément de par les liens affectifs de proximité qu'ils entretiennent avec le patient et l'entourage. Dans un contexte de charge de travail accru, devant le flou nosologique et la difficulté à évaluer l'efficacité thérapeutique, leur priorité va à la prise en charge de comorbidités lourdes, plus urgentes et à la difficile prise en charge psychosociale de la dépendance. 


\section{Références:}

1 Berr C, Vercambre MN, Akbaraly T. Epidémiologie de la maladie d'Alzheimer, approches méthodologiques et nouvelles perspectives Neuropsych Vieilliss 2009;7: 7 -14.

2. Recommandations de bonnes pratiques HAS Maladie d'Alzheimer et maladies apparentées : diagnostic et prise en charge Novembre 2011.

http://www.hassante.fr/portail/upload/docs/application/pdf/201112/recommandation_maladie d_alzheimer_et_maladies_apparentees_diagnostic_et_prsie_en_charge.pdf

3. Mura T, Dartigues JF, Berr C. How many Dementia cases in France and Europe? Alternative projections and scenarios 2010 2050, Eur Neurol 2010 ; :17 :252-59.

4. Pin Le Corre S, Somme D. Baromètre santé médecins généralistes 2009; L'accompagnement de personnes atteintes de la maladie d'Alzheimer en médecine générale. Saint Denis : inpes édition, 2011 : 205-217. (C'était sous la direction de Arnaud Gautier).

5. Alperovitch A . Vascular factors and risk of dementia: design of the Three-City Study and baseline characteristics of the study population. Neuroepidemiology. 2003; 22:316-325.

6. Helmer C, Peres K, Pariente A, et al. Primary and secondary care consultations in elderly demented individuals in France. Results from the Three-City Study. Dement Geriatr Cogn Disord 2008; 26:407-415.

7. Löppönen M, et al. Diagnosing cognitive impairment and dementia in primary health care a more active approach is needed. Age Ageing $2003 ; 32: 606-612$.

8. Olafsdotttir M, Skoog I, Marcusson J. Detection of dementia in primary care: the Linkoping study. Dement Geriatr Cogn Disord 2000;11:223-229.

9. O’Connor D, Politt P, Hyde J, Brook C, Reiss B, Roth M. Do General Practitioner miss dementia in elderly patients; BMJ 1988;297:1107-1110.

10. Bush C, Kozak j, Elmslie T. Screeningfor cognitive impairment in the elderly. Can Fam Physician 1997;43:1763-1768. 
11. Callahan C, Hendrie H, Tierney W. Documentation and evaluation of cognitive impairment in elderly primary care patients. Ann Intern Med 1996;122:422-429.

12. Bond J, Stave C, Sganga A, O’Connell B, Stanley RL. Inequalities in dementia care across Europe: key findings of the Facing Dementia Survey. Int J Clin Pract Suppl $2005 ; 46: 8-14$.

13. Wilkinson D, Sganga A, Stave C, O’ Connell B. Implications of the Facing Dementia Survey for health care professionals across Europe. Int J Clin Pract 2005, 146 Suppl : 27-31. 14. Luck T, Luppa M, Sieber J, Schomerus G, Werner P, König H-H et al. Attitudes of the German general population toward early diagnosis of dementia - Results of a Representative Telephone Survey. PLoS One $2012 ; 7: 1-3$.

15. Depraz N. Comprendre la phénoménologie. Une pratique concrète. Paris (France) : Armand Colin, 2006.

16. Depraz N, Varela FJ, Vermersch P. A l'épreuve de l'expérience. Pour une pratique phénoménologique. Bucarest (Roumanie) : Zeta Books, 2011.

17. Kaufmann JC. L'enquête et ses méthodes. L'entretien compréhensif. Paris (France): Armand Colin (collection 128): $3^{\circ}$ ed., 2011:46-58.

18. Paillé P, Mucchielli A. L'analyse qualitative en sciences humaines et sociales. Paris (France) : Armand Colin, $2^{\circ}$ ed., 2012:139-155.

19. KO Apel. Le problème de l'évidence phénoménologique in: Poulain J.Critique de la raison phénoménologique. La transformation pragmatique. Paris (France): Edition du Cerf, 1991: 37-66.

20. Tiercelin C, Thibaud P. CS Peirce Pragmatisme et pragmaticisme. Oeuvre I. Paris (France): Editions du Cerf, 2002:305-340.

21. Bourrel G. Analyse phénoménologique d'une étude sur le surpoids en médecine générale. Apport de la pragmatique. In : Recherches Qualitatives : discours théoriques et éléments 
contextuels. Montréal (Québec): Association pour la Recherche Qualitative, 2008; 6:87-103http://www.recherche-qualitative.qc.ca/hors série v6 bourrel.pdf.

22. Institut National de la Santé et de la Recherche Médicale (INSERM), Expertise collective, Maladie d'Alzheimer: Enjeux scientifiques, médicaux et sociétaux, Synthèse et recommandations. Paris : Ed de l'INSERM, 2007, 672p

23. Pentzek M, Wollny A, Wiese B, Jessen F, Haller F, Maier W et al. Apart from nihilism and stigma: what influences general practitioners' accuracy in identifying incident dementia? Am J Geriatr Psychiatry. $2009 ; 17: 965-975$.

24. Menn P, Holle R, Kunz S, Donath C, Lauterberg Y, Leidl R et al. Dementia Care in the General Practice Setting: A Cluster Randomized Trial on the Effectiveness and Cost Impact of Three Management Strategies. Value in Health. 2012; $15: 851$ - 859.

25. Haute Autorité en Santé. Rapport d'évaluation_MDC_Alzheimer-19-10-2011. in : Site de 1'HAS. www.has-sante.fr/portail/jcms/c_1118369/rapport-d-evaluation-des-medicamentsalzheimer-19-10-2011+HAS.

26. Prince M, Bryce R, Ferri C. World Alzheimer Report 201: the benefits of early diagnosis $\begin{array}{llll}\text { and } & \text { intervention. } & \text { Alzheimer's } & \text { Disease }\end{array}$ http://www.alz.co.uk/research/WorldAlzheimerReport2011.pdf

27 . Hansen E C, Hughes C, Routley G, Robinson AL. General practitioners' experiences and understandings of diagnosing dementia: Factors impacting on early diagnosis. Social Science \& Medicine. 2008; 67:1776-83.

28. Lahjibi-Paulet H, Dauffy-Alain A, Minard A, Gaxatte C, Saint-Jean O, Somme D. Attitudes toward Alzheimer's disease: a qualitative studio of the role played by social representation on a convenient sample of french general practitioners. Aging Clin Exp Res. 2012; 24: 384-390.

29. Housset E. Personne et sujet selon Husserl. Paris : PUF, collection Epiméthée, 1997, p. 16 
Tableau 1 : Guide d'entretien avec les médecins généralistes

Questionnement phénoménologique sur l'expérience vécue des troubles cognitifs

1) Quelle image vous faîtes-vous du domaine de la démence ? Selon vous, vos connaissances sur les démences vous paraissent-elles suffisantes?

2) Comment vivez-vous la prise en charge de patients déments? Ressentez-vous des difficultés pour les prendre en charge?

3) Comment abordez-vous le problème des troubles cognitifs?

4) Quelle importance attribuez-vous à ces troubles? Quelle est votre démarche personnelle ?

5) Comment vivez-vous de ne pas pouvoir initier les traitements anti-cholinestérasiques?

6) Souvenez-vous d'une expérience qui vous a marqué concernant la prise en charge de personne avec des troubles cognitifs? Que s'est-il passé? 